\title{
The induction of apoptosis in HeLa cells by the loss of LBP-p40
}

\author{
Yasufumi Kaneda ${ }^{1,4}$, Yoshinao Kaneda ${ }^{1}$, \\ Katsunari Kinoshita ${ }^{1}$, Manabu Sato ${ }^{1}$, Yoshinaga Saeki ${ }^{1}$, \\ Ryo Yamada ${ }^{2}$, Mari Wataya-Kaneda ${ }^{3}$ and Kiyoji Tanaka ${ }^{1}$ \\ ${ }^{1}$ Institute for Molecular and Cellular Biology, Osaka University, Suita, Osaka 565 \\ Japan \\ 2 Hitachi-Kasei Inc., Tsukuba, Ibaragi 300-26 Japan \\ 3 Department of Dermatology, Osaka University School of Medicine, Suita, \\ Osaka 565, Japan \\ ${ }^{4}$ corresponding author Fax: 06-877-9136; Tel: 06-879-7974. \\ e-mail: kaneday@imcb.osaka-u.ac.jp
}

Received 12.5.97; revised 1.7.97; accepted 1.8.97

Edited by Y. Kuchino

\begin{abstract}
To analyze the function of the laminin-binding protein precursor p40 (LBP-p40) in higher eukaryotic cells, plasmid DNA expressing antisense or sense CDNA for $\mathrm{p} 40$ under the control of the LacSwitch system was introduced into HeLa cells. Stable transformants were isolated, and the expression of $\mathrm{p} 40$ was assayed by Western and Northern blotting. The expression level of p40 was not affected in HeLa cell transformants cultured in $10 \%$ serum-supplemented media with the induction of antisense (AS)-p40 with 5 mM IPTG. However, both the protein and message for endogenous p40 in serum-depleted media with 5 mM IPTG were reduced to about $30-10 \%$ of the expression level in serum-free media without $5 \mathrm{mM}$ IPTG. Colony formation was inhibited with the suppression of p40. AS-p40 clones died in 7 days when cultured in serum-depleted media with $5 \mathrm{mM}$ IPTG, while clones without $5 \mathrm{mM}$ IPTG AS-p40 clones never died, even in serum-depleted media. Additionally, sense (S)-p40 clones and control CAT clones survived more than 2 weeks in serum-free media with $5 \mathrm{mM}$ IPTG. DNA fragmentation assay revealed that cell death induced by the reduction of AS-p40 resulted from apoptosis. Both the inhibition of cell growth and apoptotic cell death were partially rescued by the transfer of the p40 cDNA expression vector to AS-p40 clones. Moreover, the introduction of a synthetic hammerhead ribozyme for LBP-p40 using a fusigenic viral liposome suppressed the message for LBP-p40 even in the presence of $10 \%$ serum, and it also induced apoptosis.
\end{abstract}

Keywords: LBP-p40; antisense RNA; ribozyme; cell-growth; apoptosis

Abbreviations: LBP-p40, laminin-binding protein precursor 40 kilo-dalton protein; AS, antisense; S, sense; IPTG, isopropyl- $\beta$ $D$-thiogalactoside; CAT, chloramphenicol acetyl transferase

\section{Introduction}

A cDNA for the $40 \mathrm{kDa}$ protein was first cloned as an abundant protein in cancer cells (Yow et al, 1988; Makrides et al, 1988). The DNA sequence corresponded to the $67 \mathrm{kDa}$ laminin-binding protein first isolated from cell extracts by laminin-affinity chromatography (Rao et al, 1983; Lesot et al, 1983; Malinoff et al, 1983; Wewer et al, 1986) and was extensively investigated as a protein responsible for tumor metastasis (Liotta et al, 1986; Sobel, 1993). Therefore, it was identified as a precursor molecule of the laminin-binding protein, p40 (LBP-p40) (Yow et al, 1988). However, Northern blot analysis using p40 cDNA as a probe showed a single band of approximately 1.1-1.2 kb (Yow et al, 1988; Makrides et al, 1988; van den Ouweland et al, 1989). A recent report indicated LBP-p67 might be a dimer of LBP-p40 and that acylation might occur before or after dimerization (Landowski et al, 1995). Overproduction of LBP-p40 in BHK cells increases the production of the $67 \mathrm{kDa}$ protein at the cell surface (Wang et al, 1992). Although there is no direct evidence, it has been proposed that p40 may be a cytoplasmic precursor for LBP-p67. However, the nomenclature of the laminin-binding protein precursor generated some confusion with progress in the analysis of p40 since LBP-p40 appeared to exhibit multiple functions in various aspects of cell growth, embryonic development, and cancer progression. Several lines of evidence have indicated that the p40 is localized exclusively in the cytoplasm and is a component of $40 S$ ribosomal subunits in mammalian, Arabidopsis, and Urechis caupo cells (Auth and Brawerman, 1992; GarciaHernandez et al, 1994; Tohgo et al, 1994; Rosenthal and Wordeman, 1995). It has also been proposed that p40 is detected in developing mice retina and participates in defining the dorsal/ventral axis in developing retinas (Rabacchi et al, 1990; McCaffery et al, 1990). Wang et al. reported LBP-p40 to be the Sindvis virus receptor (Wang et al, 1992). Several mutations in Drosophila p40 resulted in a zygotic lethal by affecting oogenesis and imaginal disc development (Melnick et al, 1993). Together, these observations indicate that p40 expression is correlated with cell growth. Recently, YST1 and YST2 homologous to human LBP-p40 were cloned in Saccharomyces cerevisiae, which has no laminin (Ellis et al, 1994). The disruption of either YST1 or YST2 resulted in slow growth, probably due to reduced levels of the $40 \mathrm{~S}$ ribosome, while the complete knock-out of the YSTs was lethal (Demianova et al, 1996). This suggests that Yst proteins might be required for translation and that they contribute to assembly and/or stability of the $40 \mathrm{~S}$ ribosomal subunit, raising the questions of whether or not the reduction of LBP-p40 would affect the growth of somatic cells or higher eukaryotes and, if the reduction is lethal, what the mode of cell death would be.

We isolated a monoclonal antibody which recognized $40 \mathrm{kDa}$ protein in the nuclear envelope, cytoplasmic 
particles and perichromosomal region (Wataya-Kaneda et al, 1987), and further analysis of the antigen (Kaneda et al, 1993) indicated that the protein was associated with both chromatin DNA and nuclear matrix, and from these results we suspected that the molecule might be required for anchoring chromatin DNA to the nuclear matrix. Recently, we isolated the antigen from the cytoplasmic particles and identified it as LBP-p40. Then, we confirmed that epitopetagged LBP-p40 could be sorted to the nucleus as well as ribosome, and that the LBP-p40 was tightly attached with the nuclear matrix (Sato et al, 1996). A series of our investigations of LBP-p40 suggests that LBP-p40 may play some important role in the nucleus such as the stabilization of chromatin DNA.

No direct evidence has been presented on the function of LBP-p40, since it has not been successful to regulate the expression level of p40 in higher eukaryotic cells.

In this study, we used the LacSwitch inducible mammalian expression system (Stratagene Inc.) with lactose operon (Bourdeau et al, 1995; McCarthy et al, 1995) for the analysis of p40 gene function in mammalian cells. Stable transformants were established in HeLa cells to induce sense- or antisense-p40. Induction of antisense p40 resulted in apoptotic cell death in a serum-depleted condition in which the expression of endogenous p40 was reduced to $10-30 \%$ of the expression level without induction. DNA fragmentation assay showed that the suppression of p40 accelerated apoptotic cell death in a serum-free condition. Transient expression of exogenous p40 partially rescued apoptotic cell death. Moreover, the introduction of a synthetic ribozyme for LBP-p40 suppressed the expression of LBP-p40, even in serumsupplemented media, and induced DNA fragmentation.

\section{Results}

\section{p40 expression in inducible antisense (AS) or sense (S)-clones}

We first examined CAT gene expression in pOPI3 CAT transfectants of lacl-clone No. 15 cultured in the presence and absence of IPTG. We detected the CAT gene transcript in five out of 22 clones. Induction of the CAT message was varied. In clone No. 8 (control clone), the CAT message level induced by IPTG was highest among all the clones tested. The message was detectd at $2 \mathrm{~h}$ after the addition of $5 \mathrm{mM}$ IPTG, reached its peak level between 4 and $24 \mathrm{~h}$ and then gradually decreased (Figure 1A). The CAT message induced by IPTG was not altered by serum-depletion, and 5 mM IPTG affected neither cell growth nor total protein, RNA, or DNA synthesis (data not shown).

Next, we examined the p40 mRNA by Northern blotting using p40 cDNA as a probe. When stable transformants of AS-p40 or S-p40 were cultured in 10\% FCS-DMEM, the p40 message was not changed by IPTG (Figure 1B). We previously observed that $\mathrm{p} 40$ expression was suppressed in slow-growing cells, in cells in a confluent state, and in cells cultured in serum-depleted media, and that to the contrary, p40 expression was enhanced in cancer cells, in cells in a subconfluent state, and in cells cultured in serum-
A

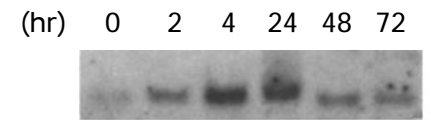

B

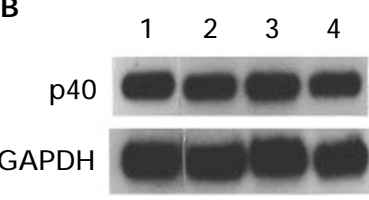

C $\begin{array}{llllllllllll}1 & 2 & 3 & 4 & 5 & 6 & 7 & 8 & 9 & 10 & 11 & 12\end{array}$

$$
\text { p40 }
$$

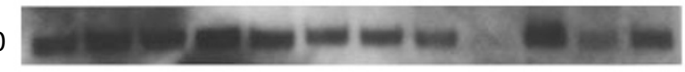

GAPDH

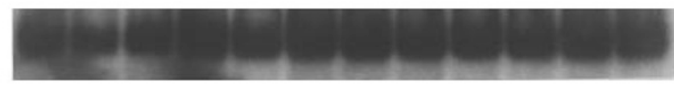

D

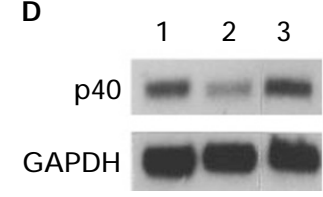

Figure 1 Gene expression in HeLa cell transformants analyzed by Northern blot. (A) CAT gene transcript induced by 5 mM IPTG was assayed by Northern blot analysis at 0, 2, 4, 24, 48 and $72 \mathrm{~h}$ after IPTG treatment. (B) Cells were cultured in 10\% FCS-DMEM with IPTG (lanes 2, 4) or without IPTG (lanes 1, 3) for 5 days). Total RNA was isolated from S-p40 clone No. 24 (lanes 1, 2), and AS-p40 clone No. 11 (lanes 3,4 ) and approximately $10 \mu \mathrm{g}$ total RNA of each clone was subjected to formalin gel electrophoresis, transferred to Hybond $\mathrm{N}^{+}$ membrane (Amersham), and hybridized with a ${ }^{32}$ P-labeled p40 or GAPDH cDNA probe. (C) Total RNA was isolated from HeLa cell clone No. 15 expressing lacl (lanes 1, 2), clone No. 8 expressing CAT (lanes 3, 4), S-p40 clone No. 18 (lanes 5, 6), S-p40 clone No. 25 (lanes 7, 8), AS-p40 clone No. 16 (lanes 9, 10) and AS-p40 clone No. 11 (lanes 11,12). Cells were treated with $5 \mathrm{mM} \mathrm{IPTG}$ (lanes 1, 3, 5, 7, 9, 11) or without IPTG (lanes 2, 4, 6, 8, 10, 12) in serum-depleted media for 6 days. Northern blot analysis was performed. (D) Total RNA was isolated from AS-p40 clone No. 11 on day 0 (lane 1), and on day 6 in serum-free media with IPTG (lane 2). Lane 3 shows the p40 transcript on day 9 in serum-free media when IPTG was removed from the culture on day 5 and the culture was continued for another 4 days in serum-depleted media

rich media. Consistent with this finding, p40 transcript in the present study was detected in cells cultured in serum-free media with and without IPTG. In a serum-free condition, p40 level in non-transfectant HeLa, or lacl-clone No. 15 and clone No. 8 expressing inducible CAT was reduced to 20$30 \%$ of each cell in $10 \%$ FCS-DMEM (data not shown). In AS-clone Nos. 11 and 16, p40 was suppressed by IPTG treatment compared with cells in media without IPTG (Figure 1C). A significant reduction (more than 50\%) of p40 mRNA was observed in three out of 40 AS-clones (Nos. 11, 16 and 33). In S-p40 clones, the p40 message was either unchanged (38 out of 40 clones) or slightly induced (two out of 40 clones) by IPTG. Especially, in ASclone No. 16, the p40 message was reduced to approximately $10 \%$ measured by an image analyzer 
(BASS 2000, Fuji). The effective clones contained a Notl insert for p40 cDNA detected by Southern blot (data not shown). Moreover, when IPTG was removed from the culture of AS-clone No. 11 on day 5 following the IPTG treatment in serum-free media, by day 9 the p40 expression level was recovered on preIPTG treatment level (Figure 1D).

Next, the p40 protein level was analyzed by Western blotting using anti-p40 polyclonal antibody. The amount of p40 was not significantly affected by the addition of $5 \mathrm{mM}$ IPTG when cultured in $10 \%$ FCS media (data not shown). However, p40 was greatly suppressed in AS-clone, Nos. 11 and 16 by the treatment of IPTG when cultured in serumfree media, whereas it was not reduced in the CAT transferred HeLa clone (Figure 2). By the densitometrical assay, the ratio of the intensity for LBP-p40 signal in the presence of IPTG to that in the absence of IPTG was about $93 \%, 26 \%$ and $12 \%$ in CAT alone No. 8, AS-p40 clone No. 11 and No. 16, respectively. Although the anti-p40 polyclonal antibody recognized the $67 \mathrm{kDa}$ protein (LBPp67; arrowhead B) as well as the $40 \mathrm{kDa}$ protein (p40; arrowhead A), the expression of LBPp67 was almost identical among clones even in the presence of IPTG. The band (approximately $44 \mathrm{kDa}$ ) pointed by an arrowhead $\mathrm{C}$ was not specific for anti-LBP-p40 antibody, since it was also detected by pre-immune rabbit serum.

\section{Viability of AS- and S-p40 transformants}

As LBP-p40 has been considered an essential protein for cellular growth, we investigated cell proliferation of AS- and Sp40 clones by labeling viable cells with tetrazolium salts. As shown in Figure 3, the growth of AS-clones decreased day by day with IPTG-treatment in serum-depleted media, while the growth of both S-clones and control clones did not decrease significantly. Additionally, when IPTG was removed on day 3

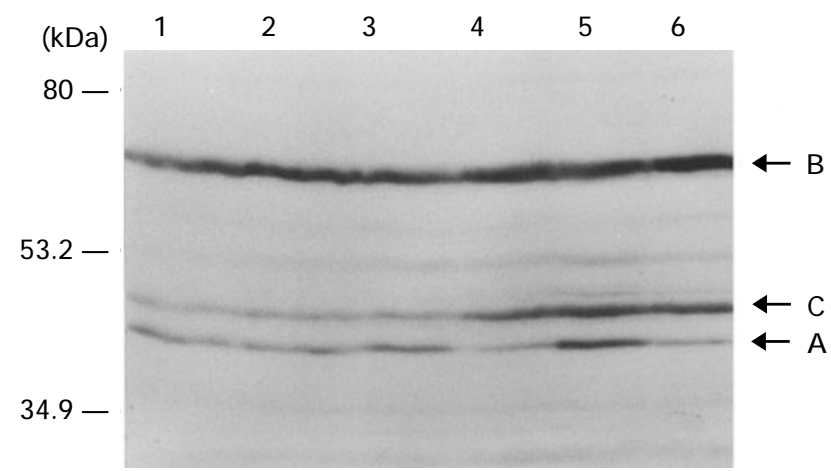

Figure 2 Western blot analysis of p40 expression in each transformant. Whole cell extract was isolated from HeLa cell clone No. 8 expressing CAT (lanes 1, 2), AS-p40 clone No. 11 (lanes 3, 4), and AS-p40 clone No. 16 (lanes $5,6)$. Cells were treated with $5 \mathrm{mM}$ IPTG (lanes 2,4 and 6 ) or without IPTG (lanes 1, 3 and 5 ) in serum-free media for 6 days before preparation of cell extract. Approximately $75 \mu \mathrm{g}$ protein was separated in SDS-polyacrylamide gel electrophoresis, transferred to nitrocellulose membrane, and blotted with antip40 rabbit polyclonal antibody followed by peroxidase-labeled anti-rabbit IgG The arrow and large arrowhead indicate LBP-p40 and LBP-p67, respectively. The small arrowhead shows a band also detected by preimmune serum from AS-clone No.11, cellular growth was not suppressed. It is suggested that cell-growth is correlated with p40 expression. For further analysis of this phenomenon, we assayed colony formation in various clones. As shown in Table 1, in AS-clone No. 16, the number of colonies formed in serum-free media with 5 mM IPTG was less than $10 \%$ of that without IPTG. In both the control clone and the S-p40 clone, under the same culture conditions, the colony formation rate exceeded $80 \%$ of that without IPTG. Even in the presence of $5 \mathrm{mM} \mathrm{IPTG}$, all the clones were alive when cultured in $10 \%$ FCS media (data not shown). In AS-clone No. 10, where the p40 mRNA was reduced by $30-40 \%$ with the IPTG treatment, the colony formation in serum-free media with IPTG was $58.4 \%$ of that without IPTG. The viability of cells appeared to be correlated with the decrease of the p40 transcript.

To examine this possibility directly, the p40 expression vector was transferred into AS-No. 11 using lipofection. As a control, the LacZ gene expression vector was also transferred to AS-clone No. 11. Seven days after transfection, the colony formation of those clones was assayed as described above. The rate of colony formation was raised from $26-56 \%$ with the transfer of p40 cDNA, while by the transfer of the LacZ gene, the rate was not significantly changed when cultured in serum-free media, with or without IPTG (Table 2). This procedure was repeated three times and similar results were obtained each time. Moreover, in AS-clone Nos. 16 and 33, colony formation was also enhanced by the transfer of the p40 cDNA expression vector (data not shown).

\section{Apoptosis in AS-p40 clones}

To analyze whether the IPTG-induced death of AS-p40 clones resulted from apoptosis, we used digoxigenin-labeled
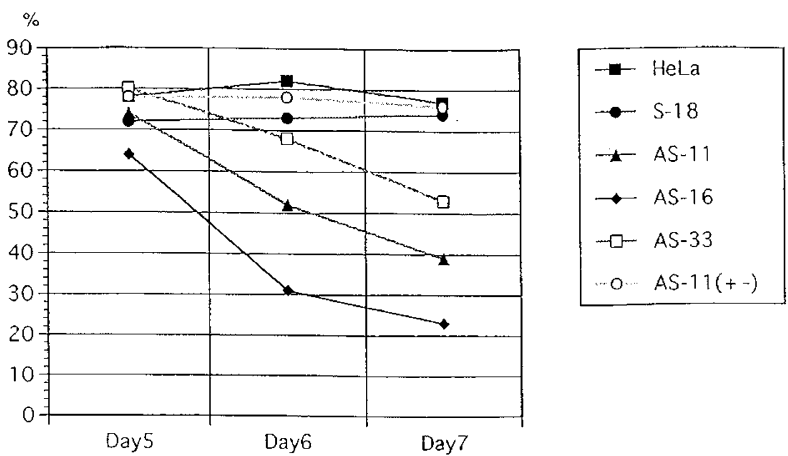

Figure 3 Cell proliferation analysis by colorimetric assay. Cells (1000) were inoculated into a 96-well plate and cultured in serum-free DMEM with or without $5 \mathrm{mM} \mathrm{IPTG}$ for 5,6 , and 7 days. At each time point, cells were labeled with MTT(3-\{4,5-dimethylthiazol-2-yl\}-2,5-diphenyl tetrazolium bromid). The absorbance at $590 \mathrm{~nm}$ of MTT formazan produced from active cells was measured using a multiwell spectrophotometer. The ordinate means the percentage of absorbance at $590 \mathrm{~nm}$ in the presence of IPTG to that in the absence of IpTG. The abscissa shows the culture period of cells in serumdepleted condition. HeLA, non-transfectant (-口-), S-p40 clone No. 18 $(--)$, AS-p40 clone No. 11 (一- -), No. 16 (一-) and No. 33 (一 $\square$-). (-O-) indicates AS-p40 clone No. 11 when $5 \mathrm{mM}$ IPTG was added to the culture in serum-free media for the initial 3 days, then removed, and the culture was continued 
Table 1 Colony formation of HeLa cells and AS-p40 clones on day 8 after culturing in serum-free DNEM with or without 5 mM IPTG

\begin{tabular}{lccc}
\hline Cell & \multicolumn{2}{c}{ Colony number } & Ratio (b)/(a) \\
HeLa, non-transfectant & (a) IPTG (-) (b) & IPTG (+) & $\%$ \\
\hline HeLa-CAT No. 8 & 326 & 368 & 86.4 \\
Sense-p40 No. 18 & 388 & 262 & 80.4 \\
Anti sense-p40 No. 10 & 332 & 376 & 96.9 \\
Anti sense-p40 No. 11 & 374 & 194 & 58.4 \\
Anti sense-p40 No. 16 & 368 & 69 & 18.4 \\
\hline
\end{tabular}

Colonies of more than 30 cells were counted after Giemsa staining. Mean colony number is presented

Table 2 Colony formation of AS-p40 clone No. 117 days after the transfer of p40 or LacZ gene in serum-depleted condition

\begin{tabular}{lcc}
\hline & \multicolumn{2}{c}{ Colony number } \\
Transferred gene & IPTG $(-)$ & IPTG (+) \\
\hline pEBc-p40 & $186(212,160)$ & $104(97,111)$ \\
pEBc-LacZ & $216(238,194)$ & $56(65,47)$ \\
\hline
\end{tabular}

Colonies of more than 30 cells counted after Giemsa staining. Mean colony number is presented with the actual number of colonies shown in parenthesis

genomic DNA with Apoptag (Figure 4), to detect apoptotic cells. Approximately $10 \%$ of the AS-No. 11 clones were positively stained with anti-digoxigenin fluorescein conjugate on day 6 after culturing in serum-free DMEM with 5 mM IPTG. The rate for Apoptag-stained cells was $5-30 \%$ in the other AS clones, Nos. 16 and 33 cultured in serum-free DMEM with $5 \mathrm{mM}$ IPTG (data not shown). The rate for the control No. 8 clone and the S-18 clone stained using the Apoptag assay kit on day 6 in serum-free DMEM with 5 mM IPTG was $1-3 \%$. When cells were cultured in serum-free DMEM without IPTG, less than $1 \%$ of the cells in each clone were apoptotic. When the media contained $10 \%$ FCS-DMEM, less than $0.1 \%$ of cells were stained by Apoptag, and the percentage was not affected by IPTG. Counter-staining by Hoechst 33258 showed that the nuclei of apoptotic cells were shrunk and condensed.

To detect the rate of cellular DNA fragmentation, BrdUlabeled DNA fragments in a cytoplasmic fraction were counted using an ELISA after culturing in serum-free DMEM with or without IPTG. Fragmented DNA increased 3.4-fold in AS-clone No. 16 in the presence of $5 \mathrm{mM}$ IPTG, compared with cells in the absence of IPTG (Table 3). In AS-clone No. 11, DNA fragmentation was slightly increased by the addition of $5 \mathrm{mM}$ IPTG. DNA fragmentation was greatly inhibited by the gene transfer of pEBc p40 to AS-p40 No. 16, but not by the transfer of pEBc-LacZ. In both the control clone No. 8 and the S-clone No. 18, DNA fragmentation was not significantly changed by IPTG treatment.

These results indicate that the loss of p40 induced apoptotic cell death. However, in the LacSwith inducible system, growth inhibition was not observed except when the AS-p40 clones were cultured in serum-free media with IPTG. We needed to determine whether the apoptotic cell death by the loss of p40 would be specific for the serumdepleted condition.

\section{Effect of the ribozyme for LBP-p40}

We suppressed the expression of LBP-p40 by transfer of the ribozyme for LBP-p40. Using the fusigenic viral-cationic liposome, an FITC-labeled wild-type ribozyme was introduced into $100 \%$ of the HeLa cells; fluorescence was exclusively present in the cytoplasm, and faded out in 3-5 days. We synthesized three different 38 mer ribozymes for LBP-p40, all of which digested a 29-mer targeted sequence of LBP-p40 in vitro (data not shown). However, when introduced into the HeLa cells by the liposome, one ribozyme, No. 111, recognized the GUC sequence of LBP-p40 mRNA at Nos. 220 to 222 nucleotides was the most effective for suppressing the LBP-p40 message. By the use of this ribozyme, the transcript for LBP-p40 was decreased day by day even in the media supplemented with $10 \%$ FCS and reduced to less than $10 \%$ of cells treated with HIV-Tat ribozyme on day 4 and 6 , while the human GAPDH transcript was not significantly reduced by No. 111 ribozyme (Figure 5A). The digested RNA by the ribozyme was not detected in the HeLa cell extract using Northern blot, although those ribozyme cleaved synthesized p40 substrates into two fragments when mixed in a tube (data not shown). By the introduction of LBP-p40 ribozyme No. 111, fragmented DNA of HeLa cells cultured in $10 \%$ FCS-DMEM increased between day 4 and 6 , while DNA fragmentation was not induced with the control Tat ribozyme (Figure $5 \mathrm{~B}$ ). We evaluated apoptosis by counting nick-end labeled cells (Figure $5 \mathrm{C}$ ). With the introduction of LBP-p40 ribozyme No. 111, of more than 500 cells observed, the number of nick-end labeled cells was approximately $15 \%$ on day 4 and gradually increased to $22 \%$ on day 6 . Neither the Tat ribozyme nor the empty HVJ-cationic liposome generated more than $3 \%$ nick-end labeled cells.

\section{Discussion}

This study shows that apoptotic cell death is correlated with the reduction of $\mathrm{p} 40$, and that the transient expression of exogenous p40 partially rescued this phenomenon. This finding seems to be consistent with previous reports on LBPp40 indicating the relationship between p40 and cellular growth (Makrides et al, 1988; Rabacchi et al, 1990; McCaffery et al, 1990; Melnick et al, 1993; Ellis et al, 1994). However, no previous reports have documented apoptotic cell death by the suppression of $\mathrm{p} 40$. In this respect, our induction of apoptosis in As-p40 clones may indicate a novel function of LBP-p40 in higher eukariotic cells.

In our first trial, the expression of p40 was suppressed by the induction of antisense-p40 by $5 \mathrm{mM}$ IPTG in serumfree media. Therefore, we deliberately controlled for the influences of IPTG and serum withdrawal to show the effect of the loss of p40. CAT-expressing clones under the control of IPTG in serum-free media neither inhibited growth nor induced apoptosis, and $5 \mathrm{mM}$ IPTG was not toxic to cells either in a serum-free or serum-rich condition. Among ASp40 clones, the inhibition of colony formation was well correlated with the rate of $\mathrm{p} 40$ suppression. Finally, by the transfer of exogenous p40 driven by a CMV promotor, both colony formation and apoptosis were partially recovered. From these results, we conclude that both the inhibition of 
cell-viability and the induction of apoptosis resulted from the loss of p40, not from IPTG treatment in serum depletion. The expression of endogenous p40 was reduced to $20-30 \%$ in a serum-depleted condition compared with the level in serum-supplemented media. The change of p40 expression by S-or As-p40 was not detected unless serum was depleted. In other words, the expression of p40 in serum-rich media was too abundant to
A

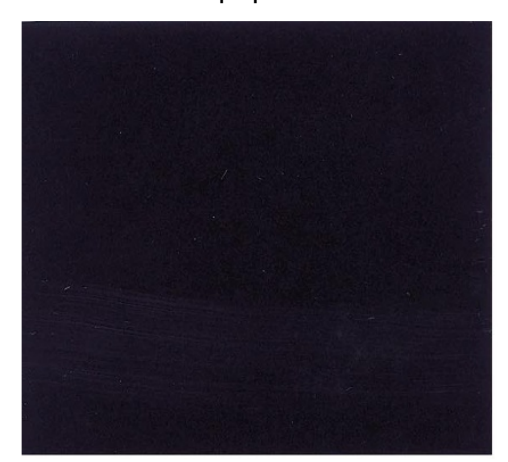

B

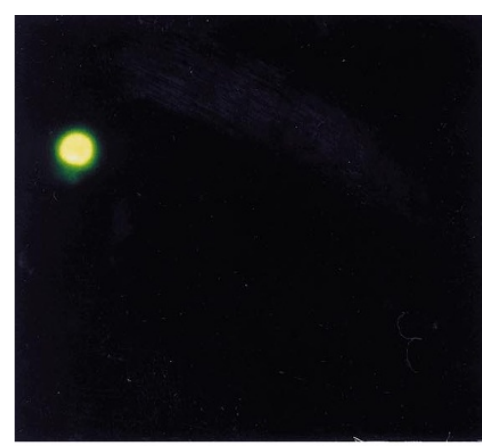

C

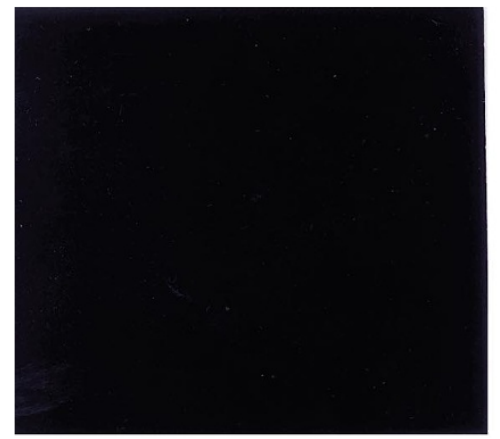

D

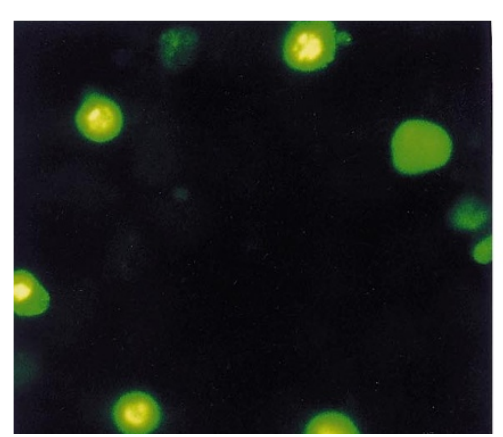

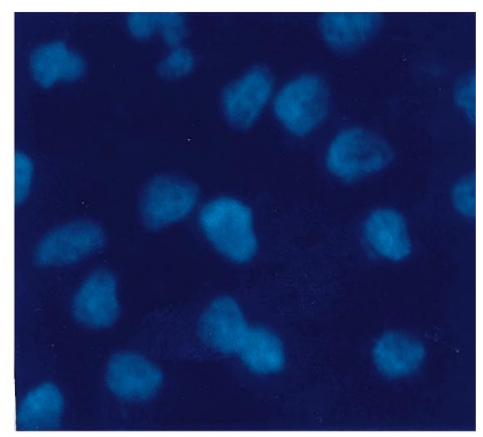

Nuclei
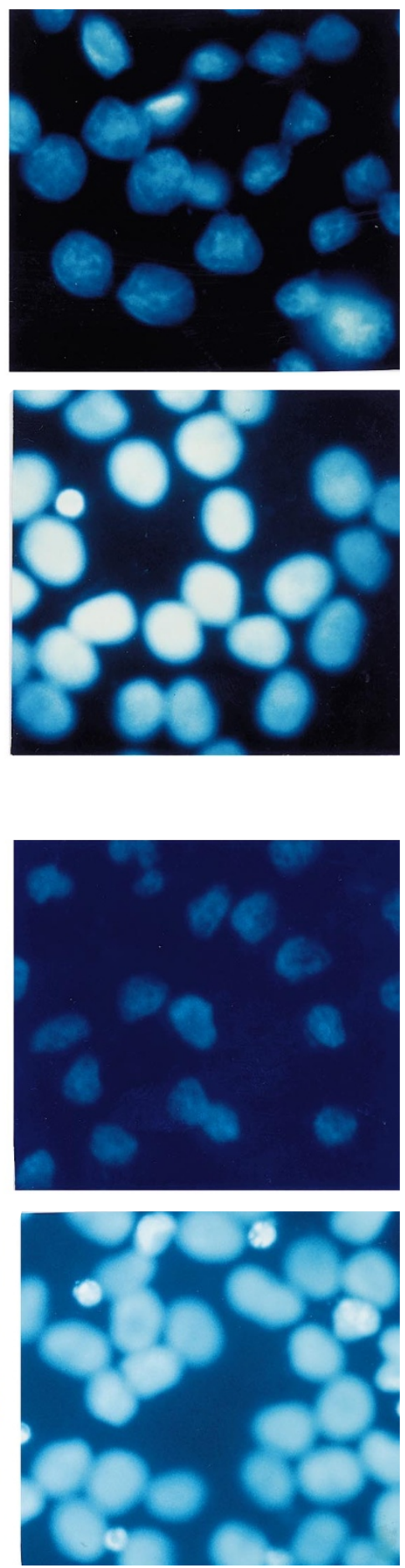

Figure 4 Apoptosis in each transformant. Apoptotic cells in HeLa-CAT clone No. 8 (A, B) or AS-p40 clone No. 11 (C, D) were detected by anti-digoxigenin fluorescein conjugate (Apoptag) on day 6 after culturing cells in serum-depleted media with (B, D) or without (A, C) 5 mM IPTG. Nuclei are stained with Hoechst 33258. Focus is adjusted on the condensed nuclei 
Table 3 Apoptosis evaluated by cellular DNA fragmentation ELISA

\begin{tabular}{lcc}
\hline & \multicolumn{2}{c}{ Absorbance at $450 \mathbf{~ n m}$} \\
Cell & IPTG (-) & IPTG (+) \\
\hline HeLa-CAT No. 8 & $0.064(0.010)$ & $0.069(0.019)$ \\
Sense-p40 No. 18 & $0.081(0.016)$ & $0.081(0.020)$ \\
Anti sense-p40 No. 11 & $0.068(0.006)$ & $0.084(0.003)$ \\
Anti sense-p40 No. 16 & $0.065(0.003)$ & $0.223(0.014)$ \\
Anti sense-p40 No. 16+pEBc-p40 & $0.070(0.005)$ & $0.118(0.007)$ \\
Anti sense-p40 No. 16+pEBc-LacZ & $0.068(0.006)$ & $0.215(0.006)$ \\
\hline
\end{tabular}

Cells labeled with BrdU were cultured in serum-free media with or without IPTG and fragmented DNA in the cytoplasm was measured by the absorbance at $450 \mathrm{~nm}$ on day 6 after labeling. Values in parentheses indicate standard deviation. AS-p40 No. 16+pEBc-p40 (or pEBc-LacZ) means AS-p40 clone No. 16 transfected with p40 expression vector, pEBc-p40 (or LacZ expression vector, pEBc-LacZ) by lipofection 1 day before IPTG treatment

be decreased by AS RNA. However, the ribozyme was more powerful for reduction of the targeted gene expression. Using our fusigenic viral liposome, ribozymes as well as AS-oligodeoxynucleotides can be directly introduced into the cytoplasm without degradation (Kitajima et al, 1997). It is estimated that more than a half-million molecules of oligonucleotides can be introduced into one cell by this delivery system (Dzau et al, 1996). Therefore, both the suppression of $\mathrm{p} 40$ and DNA fragmentation were achieved by the ribozyme delivery even in serumsupplemented condition. This also confirms apoptosis was induced by the loss of $\mathrm{p} 40$.

Although no direct evidence has yet been elucidated, LBP-p40 is considered to be a precursor of LBP-p67 (Landowski et al, 1995). Western blotting showed that p40 was decreased by the induction of AS-p40, while the antisense did not affect the expression of p67 (Figure 2). Although this does not give us direct evidence of the relationship between these two molecules, it is unlikely that apoptotic cell death resulted from the expression level of LBP-p67.

The mechanism of apoptotic cell death by the loss of p40 remains to be elucidated. It has been suggested that LBP-p40 is required for translation in plants (GarciaHernandez et al, 1994), lower eukaryotes (Rosenthal and Wordeman, 1995; Demianova et al, 1996) and presumably in higher eukaryotes (Auth and Brawerman, 1992; Tohgo et al, 1994). The rate of protein synthesis in AS-p40 clones was assayed by ${ }^{3} \mathrm{H}$-leucine incorporation. However, no significant reduction was observed in AS-p40 clones compared with that in control CAT clones (data not shown). Therefore, it is unlikely that apoptosis was induced by the suppression of net protein synthesis. It may be supposed that $\mathrm{p} 40$ is required for the translation of some specific molecules. When the expression of bcl-2 in AS-p40 clones was analyzed by Western blotting, the expression level of bcl-2 was almost comparable with that in control CAT clones (data not shown). As described in Introduction section, we reached LBP-p40 from the analysis of chromosomal proteins, and have anticipated that LBP-p40 might be an anchoring protein to stabilize chromatin DNA in the nucleus (Wataya-Kaneda et al, 1987; Kaneda et al, 1993; Sato et al, 1996). Recently, we discovered that recombinant $\mathrm{LBP}=\mathrm{p} 40$ tightly bound to DNA cellulose column through the association with core histones (unpublished observation), and we also succeeded in coprecipitation of nuclear matrix proteins with LBP-p40. We recently found that AS-p40 clones were arrested at the $S$ phase of the cell cycle (unpublished data). Therefore, taken together, we speculate that chromatin DNA may become unstable by the loss of LBP-p40 as a chromatin anchoring protein and that the unstable chromatin DNA might be liable to nuclease attack during cell-cycle arrest. Although much more extensive studies are required to elucidate the relationship between apoptosis by the loss of LBP-p40 and S-phase arrest, since LBP-p40 is an essential protein in higher eukaryotic cells, the reduction by the ribozyme of LBP-p40 in cancers may lead to a new approach for cancer therapy.

\section{Materials and Methods}

\section{Plasmid DNA construction}

The LacSwitch inducible mammalian expression system (Stratagene Inc.) consisted of p3'SS containing a lacl gene and pOPI3CAT containing a lac operator. Human LBP-p40 cDNA, isolated from a HeLa cell cDNA library, kindly provided by $\mathrm{Dr} H$ Nojima, Osaka University, and Notl linker was ligated at $5^{\prime}$ and $3^{\prime}$ termini of the cDNA. The pOPI3 sense or antisense-p40 contained right- or reverseoriented entire sequence for p40 cDNA at Notl sites of the pOPI3CAT plasmid, respectively. All plasmid DNAs were purified by cesium chloride ultracentrifugation.

\section{Isolation of transformants}

We transferred $20 \mu \mathrm{g}$ of $\mathrm{p} 3^{\prime} S \mathrm{~S}$ to $10^{6} \mathrm{HeLa}$ cells by the calciumphosphate precipitation method, and cloned 30 stable transformants after a 2-week selection by hygromycin B $(300 \mu \mathrm{g} / \mathrm{ml})$. The nuclear expression of lacl was examined by both protein blotting and indirect immunofluorescence using rabbit polyclonal antibody against recombinant lacl (Statagene Inc.). Of the clones isolated, clone No. 15 expressed the highest level of lacl protein, and all the No. 15 cells showed clear nuclear staining by indirect immunofluorescence using the anti-lacl antibody. Using a calcium-phosphate precipitation method, $20 \mu \mathrm{g}$ of pOPI3 sense or antisense-p40 was transferred to $10^{6}$ cells of clone No. 15. As a control, pOPI CAT was transferred to cells of clone No. 15 by the same method. Stable transformants were cloned by the selection of $900 \mu \mathrm{g} / \mathrm{ml}$ geneticin (G418, Gibco BRL) in each case.

\section{Cell culture}

HeLa cells were maintained in Dulbecco's modified Eagle minimum essential medium (DMEM) supplemented with $10 \%$ fetal calf serum (FCS). To induce sense or antisense-p40 in the LacSwitch induction system, cells were cultured in 10\% FCS-DMEM or serum-depleted DMEM containing $5 \mathrm{mM}$ IPTG. Fresh medium with or without IPTG was supplemented every 2 days.

\section{Northern and Western blotting}

Total RNA from each clone was isolated using Isogen (Nippon Gene, Inc.), and an equal amount of the RNA $(8-15 \mu \mathrm{g})$ was separated in 
formalin gel and subjected to Northern blotting using ${ }^{32} \mathrm{P}$-labeled cDNA for LBP-p40 or glyceraldehyde-3-phosphate dehydrogenase (GAPDH, Clontech) or chloramphenicol acetyltransferase (CAT) as a probe. The intensity of each signal was measured by an image analyzer (BASS 2000, Fuji). For protein analysis, cells subconfluent in a $60 \mathrm{~mm}$ dish were harvested and lysed in modified RIPA lysis buffer

\section{A}

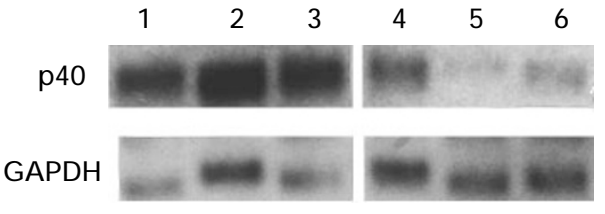

B
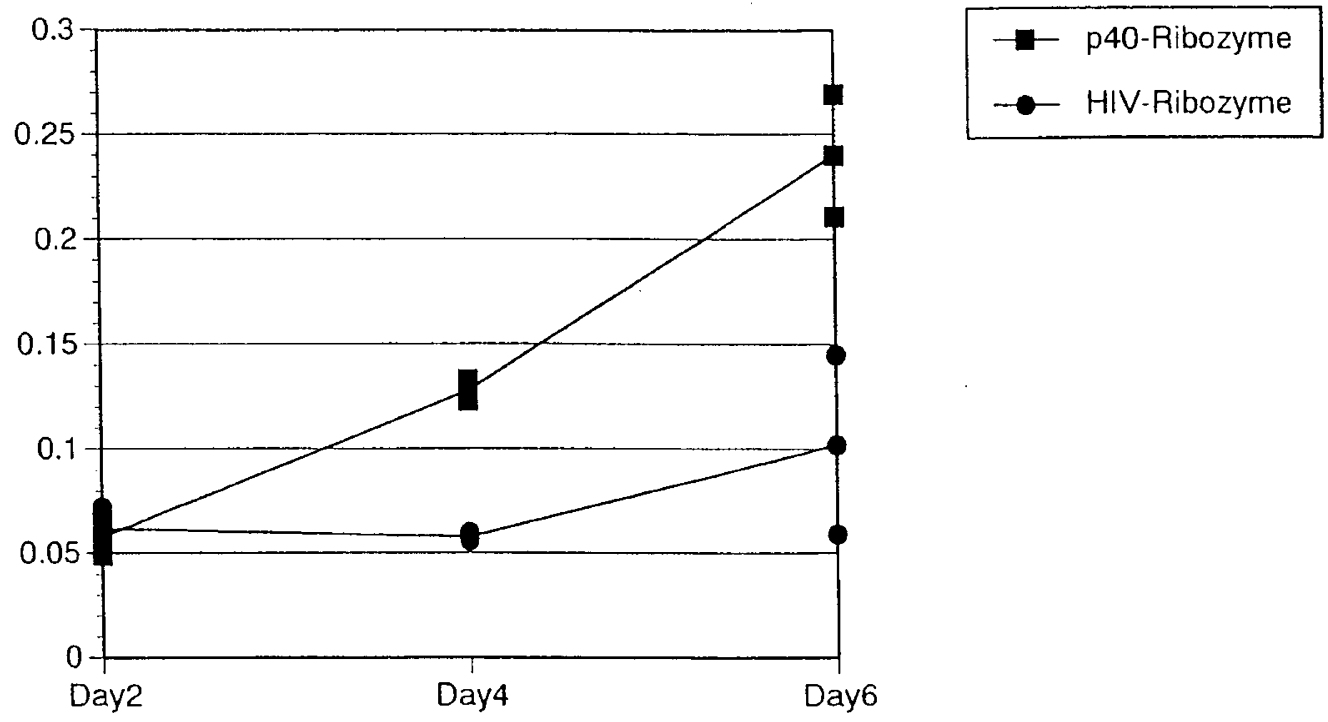

C
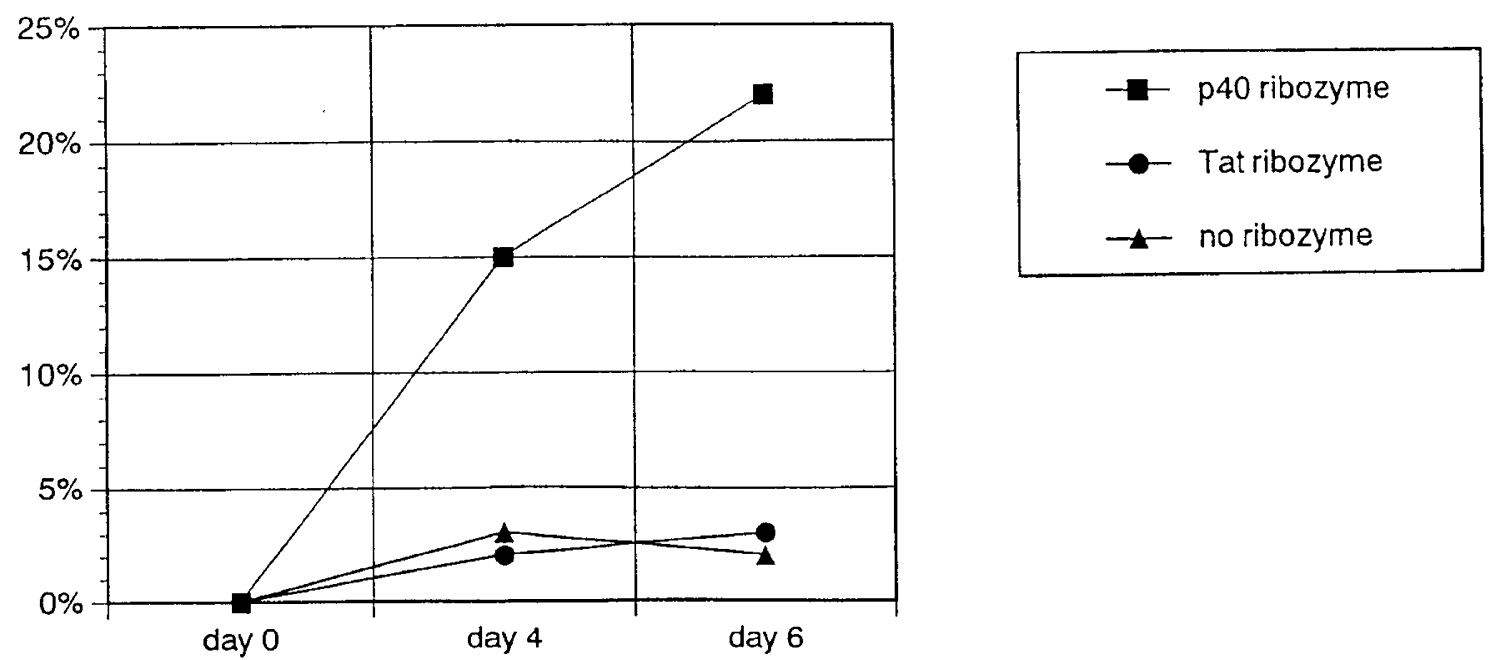

Figure 5 Effects of the ribozyme for LBP-p40. (A) The transcript for LBP-p40 was detectred by Northern blot analysis. Total RNA of HeLa cells treated with fusigenic viral-cationic liposomes containing HIV-TAT ribozyme (lanes 1, 2 and 3) or LBP-p40 ribozyme No. 111 (lanes 4, 5 and 6 ) was subjected to Northern blot analysis on day 2 (lanes 1 and 4), day 4 (lanes 2 and 5), and day 6 (lanes 3 and 6) after the ribozyme transfer. Human GAPDH was also probed as an internal control. (B) DNA fragmentation of HeLa cells introduced with HIV-Tat ribozyme (- -) or LBP-p40 ribozyme No. 111 (-口-) by fusigenic viral-cationic liposome was assayed on day 2, 4 and 6 after the transfer. The ordinate indicates the absorbance at $450 \mathrm{~nm}$. The abscissa shows the period of cells cultured in $10 \%$ FCSDMEM after ribozyme transfer. (C) The nick-end labeled HeLa-cells detected by Apoptag (colonies of more than 500 cells) were microscopically observed on day 0 ,

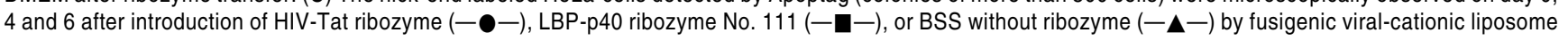


(50 mM Tris- $\mathrm{Cl} \mathrm{pH} 8,150 \mathrm{mM} \mathrm{NaCl}, 1 \%$ NP40, $0.1 \%$ SDS, $0.5 \%$ deoxycholate, $5 \mathrm{mM}$ EDTA) containing $2 \mathrm{mM}$ phenylmethylsulfonylfluoride. Supernatant was obtained after centrifugation $(12000 \mathrm{~g}$, $10 \mathrm{~min}$ ), and the amount of protein was measured by bicinchoninic acid assays (Pierce). Approximately $75 \mu \mathrm{g}$ of protein was subjected to Western blotting, and the signal was detected using anti-p40 polyclonal antibody followed by a horseradish peroxidase-conjugated second antibody and visualized by chemiluminescence reagent (ECL, Amersham Inc.). The intensity of each signal was measured by a densitometer (Becton-Dickinson).

\section{Cell viability assay}

Approximately $10^{4}$ cells were inoculated into each well of 6-well plates (Corning) and cultured for 1 day in 10\% FCS-DMEM. The medium was then changed to serum-depleted DMEM with or without $5 \mathrm{mM} \mathrm{IPTG.}$ After fixing with cold $80 \%$ methanol and staining with $5 \%$ Giemsa, the number of colonies exceeding 30 cells was counted. To evaluate the cell-growing conditions, $10^{3}$ cells inoculated into 96-well plates (Corning) were cultured in serum-free DMEM with and without $5 \mathrm{mM}$ IPTG for 5, 6, and 7 days. At each time point, cells were labeled with MTT(3-\{4,5-dimethylthiazol-2-yl\}-2,5-diphenyl tetrazolium bromid). The absorbance at $590 \mathrm{~nm}$ of MTT formazan produced from active cells was measured using amultiwell spectrophotometer with an MTT assay kit (Boehringer). In each case, values recorded were the average of triplicate readings.

\section{Apoptosis assay}

Cells on glass slides were fixed with $4 \%$ paraformaldehyde $(\mathrm{pH} \mathrm{7.4)}$ and treated with terminal deoxynucleotidyl transferase. Apoptotic cells were visualized with an anti-digoxigenin fluorescein conjugate using an Apoptag Plus kit (Oncor). To quantify apoptosis, $3 \times 10^{3}$ cells were inoculated onto 96-well-plates and DNA fragmentation was assayed by ELISA using a Cellular DNA Fragmentation ELISA kit (Boehringer Mannheim). Values used were the average of triplicate assays.

\section{Gene transfer of $\mathrm{p} 40$ expression vector}

The full length of $p 40$ cDNA was cloned in our original $p E B c$ vector, which contained a cytomegalovirus promotor to drive an inserted cDNA and Epstein-Barr virus replicon apparatus (Ori P and EBNA-1 sequence) for autonomous replication of the plasmid. We transferred $8 \mu \mathrm{g}$ of pEBc p40 into $5 \times 10^{5}$ cells using LipofectAmine (Gibco BRL). As a control, LacZ gene was cloned in our $\mathrm{pEBc}$ vector and transferred into cells as described above. Judging from the LacZ gene expression by X-gal staining, $20-40 \%$ of the cells were transiently transduced from experiment to experiment under this transfection condition, and the gene expression level did not decline for more than 1 week when our $\mathrm{pEBc}$ vector was used.

\section{Riboztme transfer}

Hammerhead ribozymes for LBP-p40 were synthesized by HitachiKasei Inc. (Tsukuba, Japan). We entrapped $50 \mu \mathrm{g}$ ribozyme in liposome consisting of phosphatidylcholine, cholesterol, and $3 \beta[\mathrm{N}$ ( $\mathrm{N}^{\prime}, \mathrm{N}^{\prime}$-dimethylaminoethane)-carbamoyl]cholesterol(DC-Chol), and the liposome was fused with UV-inactivated HVJ (Hemagglutinating Virus of Japan; Sendai virus) to form the fusigenic viral-cationic liposome (Saeki et al; manuscript submitted), which was $10-100$ times more effective in the transfer of genes to cultured cells than our former fusigenic viral anionic liposome (Dzau et al, 1996). HeLa cells were incubated with the liposome at $37^{\circ} \mathrm{C}$ to introduce the ribozyme. Using the fusigenic viral-cationic liposome, fluorescence-labeled ribozyme was introduced into the cytoplasm of all the HeLa cells (unpublished observation). The ribozyme for the Tat protein of human immunodeficiency virus (HIV) was used for negative control. Northern blotting and a DNA fragmentation assay were carried out as described above.

\section{References}

Auth D and Brawerman G (1992) A 33kDa polypeptide with homology to the laminin receptor; component of translation machinery. Proc. Natl. Acad. Sci. (USA) 89: $4368-4372$

Bourdeau N, Sympson CJ, Werb Z and Bissell MJ (1995) Suppression of ICE and apoptosis in mammary epitherial cells by extracellular matrix. Science 267 : $891-893$

Demianova M, Formosa TG and Ellis SR (1996) Yeast proteins related to the p40/ laminin receptor precursor are essential components of the $40 \mathrm{~S}$ ribosomal subunit. J. Biol. Chem. 271: 11383-11391

Dzau VJ, Mann MJ, Morishita R and Kaneda Y (1996) Fusigenic viral liposome for gene therapy in cardiovascular diseases. Proc. Natl. Acad. Sci. (USA) 92: $11421-11425$

Ellis S, Miles J and Formosa TG (1994) Characterization of NAB1, a yeast gene coding for a protein homologous to the mammalian $67-\mathrm{kDa}$ laminin receptor. FASEB J. 8: A1312

Garcia-Hernandez M, Davies E and Staswick PE (1994) Arabidopsis p40 homologue. J. Biol. Chem. 269: 20744-20749

Kaneda Y, Kinoshita K, Sato M, Tanaka K and Kaneda Y (1993) The analysis of 40kDa protein, p40, in interphase cells and mitotic cells. J. Cell Sci. 106: $741-$ 748

Kitajima I, Hanyu N, Soejima Y, Hirano R, Arahira S, Yamaoka S, Yamada R, Maruyama I and Kaneda $Y$ (1997) Efficient transfer of synthetic ribozymes into cells using hemagglutinating virus of Japan (HVJ)-Cationic liposomes. J. Biol. Chem. 272: 27099-27106

Landowski TH, Dratz EA and Starkey JR (1995) Studies of the structure of the metastasis-associated $67 \mathrm{kDa}$ laminin binding protein; fatty acid acylation and evidence supporting dimerization of the $32 \mathrm{kDa}$ gene product to form the mature protein. Biochemistry 34: 11276-11287

Lesot H, Kuhl U and von der Mark K (1983) Isolation of a laminin binding protein from muscle cell membrane. EMBO J. 2: 861-865

Liotta LA, Rao CN and Wewer UM (1986) Biochemical interactions of tumor cells with the basement membrane. Ann. Rev. Biochem. 55: 1037-1057

Makrides SS, Chitpatima T, Bandyopadhyay R and Brawerman G (1988) Nucleotide sequence for a major messenger RNA for a 40 killodalton polypeptide that is under translational control in mouse tumor cells. Nucleic Acid Res. 16: 2349

Malinoff HL and Wicha MS (1983) Isolation of a cell surface receptor protein for laminin from murine fibrosarcoma cells. J. Cell Biol. 96: 1475-1479

McCaffery P, Neve RL and Drager UC (1990) A dorso-ventral asymmetry in the embryonic retina defined by protein conformation. Proc. Natl. Acad. Sci. (USA) 87: $8570-8574$

McCarthy SA, Samuels ML, Pritchard CA, Abraham JA and McMahon M (1995) Rapid induction of heparin-binding epidermal growth factor/diptheria toxin receptor expression by raf and ras oncogenes. Genes \& Development 9: 1953-1964

Melnick MB, Noll E and Perrimon N (1993) The Drosophila stubarista phenotype is associated with a dosage effect of the putative ribosome-associated protein Dp40 on spineless. Genetics 135: 553-564

Rabacchi SA, Neve RL and Drager UC (1990) A positional marker for the dorsal embryonic retina is homologous to the high-affinity laminin receptor. Development 109: 521-531

Rao NC, Barsky SH, Terranova VP and Liotta LA (1983) Isolation of tumor cell laminin receptor. Biochem. Biophys. Res. Comm. 111: 804-808

Rosenthal ET and Wordeman L (1995) A protein similar to the $67 \mathrm{kDa}$ laminin binding protein and p40 is probably a component of the translational machinery in Urechis caupo oocytes and embryos. J. Cell Sci. 108: 245-256

Sato M, Kinoshita K, Kaneda Y, Saeki Y, Iwamatsu A, Tanaka Kand Kaneda Y (1996) Analysis of nuclear localization of laminin binding protein precursor p40 (LBP) p40). Biochem. Biophys. Res. Comm. 229: 896-901 
Sobel ME (1993) Differnetial expression of the $67 \mathrm{dDa}$ laminin receptor in cancer. Semin. Cancer Biol. 4: 311-317

Tohgo A, Takasawa S, Munakata H, Yonekura H, Hayashi N and Okamoto H (1994) Structural determination and characterization of a $40 \mathrm{kDa}$ protein isolated from rat 40S ribosomal subunit. FEBS Lett. 340: 133-138

van ven Ouweland AMW, van Duijnhoven HLP, Deichman KA, van Groningen JJM, de Leij L, and van de Ven WJM (1989) Characteristics of a multicopy gene family predominantly consisting of processed pseudogenes. Nucleic Acid Res. 16: $3829-3843$

Wang K-S, Kuhn RJ, Strauss EG, Ou S and Strauss JH (1992) High-affinity laminin receptor is a receptor for Sindbis virus in mammalian cells. J. Virol. 66: 49925001
Wataya-Kaneda M, Kaneda Y, Sakurai T, Sugawa H and Uchida T (1987) A monoclonal antibody against the nucleus reveals the presence of a common protein in the nuclear envelope, the perichromosomal region, and cytoplasmic vesicles. J. Cell Biol. 104: 1-7

Wewer UM, Liotta LA, Jaye M, Ricca GA, Drohan WN, Claysmith AP, Rao CN, Wirth P, Coligan JE, Albrachtsen R, Murdryj M and Sobel ME (1986) Altered levels of laminin receptor $\mathrm{mRNA}$ in various human carcinoma cells that have different abilities to bind laminin. Proc. Natl. Acad. Sci. (USA) 83: 7137-7141

Yow HK, Wong JM, Chen HS, Lee CG, Davis S, Steele Jr GB and Chen LB (1988) Increased mRNA expression of a laminin-binding protein in human colon carcinoma; complete sequence of a full-length cDNA encoding the protein. Proc. Natl. Acad. Sci. (USA) 85: 6394-6398 